\title{
Données expérimentales sur le fluage du gypse saccharoïde en condition saturée
}

\section{MOIRIAT}

BRGM-SAR Océan Indien 5, rue Sainte-Anne, BP 906 97478 Saint-Denis (Réunion) Cedex d.moiriat@brgm.fr

\section{P. POTHERAT}

CETE de Lyon 25, avenue F.-Mitterrand case 1

69674 Bron Cedex pierre.potherat@equipement. gouv.fr

\section{E. MASSIEU}

LCPC Paris

Service physico-chimie des matériaux

58, boulevard Lefebvre 75732 Paris Cedex 15 etienne.massieu@lcpc.fr

\section{J.-L. DURVILLE}

Ministère de l'Équipement CGPC, $3^{\mathrm{e}}$ section

Tour Pascal-B,

92055 Paris-la Défense jean-louis.durville@ equipement.gouv.fr

Le fluage est un des phénomènes majeurs à l'origine de dégradations dans les anciennes exploitations souterraines de gypse de la région parisienne. Afin de mieux appréhender les mécanismes en œuvre lors du fluage de ce matériau, des essais par paliers et à charge constante, ont été menés en condition saturée et sans pression de confinement sur deux types de gypse saccharoïde.

Après un stade de fluage transitoire, le fluage dit stationnaire se caractérise par une relation pseudolinéaire entre la déformation et le temps, et est asymptotique pour des valeurs de charge inférieure à la limite élastique du gypse. Pour des valeurs de charge supérieure à la limite élastique du gypse, la vitesse de déformation axiale tend vers une valeur minimale au-delà de laquelle démarre le stade court du fluage accéléré menant à la ruine de l'éprouvette. Le mécanisme de rupture dans ces conditions expérimentales se caractérise par une décohésion des grains puis par une microfissuration croissante. A la transition fluage stationnaire-fluage accéléré, cette fissuration devient observable macroscopiquement sur l'éprouvette, on passe alors d'un mode de déformation continu à un mode discontinu.

Mots-clés : carrière souterraine, gypse saccharroïde. fluage, fluage stationnaire, limite élastique, endommagement.

\section{Experimental data on creep of the saccharoid gypsum in saturated condition}

Creep is one of the major phenomena at the origin of damages in the old gypsum underground mines of the Paris area. In order to better apprehend the mechanisms involved it during the creep of this material, creep tests with a constant load or several loading, were carried out in saturated condition and without confining pressure on two types of saccharoid gypsum. After a transitory stage of creep, the secondary stage or steady state, is characterized by a pseudo linear relation between the axial strain and time, and is asymptotic for values of loads lower than elastic boundary stress of the gypsum. For load values higher than the elastic boundary stress, the axial strain rate tends towards a minimal value beyond which starts the tertiary stage of creep which leads quickly to the breaking-point. The mechanism of damaging under these experimental conditions is characterized by a ungluing of the grains and increasing microcracking. That increase in relative porosities, gradual during the steady state, then begin the tertiary stage of creep. 


\section{1}

\section{Introduction}

En région parisienne, les carrières souterraines abandonnées de gypse ludien, exploitées par chambres et piliers, montrent généralement un stade de dégradation beaucoup plus avancé que celui des exploitations du même âge, de craie ou de calcaire massif. Cela est dû en grande partie au fluage en condition saturée dans les piliers de cette roche monominérale (Durville et al., 1993).

Si l'importance de ce phénomène pour le gypse a déjà été bien développée expérimentalement (Auvray et al., 2004), et modélisée (Hoxha et al., 2005) grâce notamment aux analogies ente le sel gemme et le gypse, les mécanismes d'endommagement du gypse mis en œuvre lors de son fluage en condition saturée, à faible pression et à faible température, restent cependant à comprendre, et plus précisément lors de la transition fluage stationnaire-fluage accéléré. Cette étude se propose, par le biais d'essais de fluage, de préciser le comportement du gypse saccharoïde sous charge constante et en condition saturée. Deux gypses cristallins provenant des buttes de l'Hautil et de Montmorency en région parisienne ont été testés.

2

\section{Caractéristiques du gypse étudié}

Le faciès saccharoïde du gypse présente les caractéristiques d'un dépôt évaporitique par sédimentation et accrétion de germes cristallisés à partir d'eaux sursaturées. Il s'agit d'un assemblage de cristaux lenticulaires accolés les uns aux autres et soulignant un litage plus ou moins exprimé suivant les conditions de dépôt (anisotropie). Bien qu'il existe des cristaux typiques avec macles en fer de lance ou queue d'aronde, les cristaux en individus isolés ou simplement juxtaposés forment l'essentiel des dépôts dans les plâtrières de la région parisienne (Deicha, 1974).

Les dépôts d'âge ludien se répartissent en strates: les cristaux montrent une grande similitude en taille au sein d'une même strate, et de grandes variations de taille d'une strate à l'autre. Des prélèvements effectués dans une carrière souterraine creusée sous la butte de Montmorency dans le Val-d'Oise ont conduit à distinguer trois classes principales de gypse saccharoïde en fonction de la taille movenne des cristaux (d), comprise entre la dizaine de microns et le centimètre (Potherat et al., 1999):

- gypse macrogrenu noté $\mathrm{Mg}: \quad \mathrm{d} \geq 1 \mathrm{~mm}$

- gypse grenu (ou macrocristallin)

noté $m g$ :

$0,1 \mathrm{~mm} \leq \mathrm{d}<1 \mathrm{~mm}$

- gypse microgrenu

$\mathrm{d}<0,1 \mathrm{~mm}$

(ou microcristallin) noté $\mu g$ :

Cette étude préalable (Potherat et al., 1999) a par ailleurs confirmé qu'avec l'augmentation de la taille de grain, la porosité augmente et les caractéristiques mécaniques diminuent.

Trois types de gypse sont étudiés en fluage, un faciès mg (gypse V1), un faciès $\mu g$ (gypse V2B) ainsi qu'un faciès intermédiaire entre mg et $\mu \mathrm{g}$ (gypse V2A). Ces trois faciès ont déjà été caractérisés pétrographiquement et mécaniquement (Durville et al., 1993;
Potherat et al., 1999), Pour des éprouvettes d'un diamètre de $40 \mathrm{~mm}$ et d'une longueur de $80 \mathrm{~mm}$ (élancement 2), taillées perpendiculairement au litage, quelques caractéristiques moyennes de ces matériaux figurant dans le tableau I.

TABLEANI Caractéristiques du gypse étudié en fluage avec n la porosité (\%), $V_{\text {tw }}$ la vitesse des ondes longitudinales mesurée en condition saturée $(\mathrm{m} / \mathrm{s})$ et $R_{\text {cu }}$ la résistance moyenne à la compression simple mesurée en condition saturée (MPa).

Characteristics of the gypsum studied in creep with n porosity (\%), $\mathrm{V}$, the velocity of longitudinal waves measured in saturated condition (m/s) and $R_{\text {the uniaxial compressive mean strength }}$ measured in saturated condition (MPa).

\begin{tabular}{lccc} 
Type & $\begin{array}{c}\mathrm{B} \\
(\%)\end{array}$ & $\begin{array}{c}\mathrm{V} / \mathrm{s} \\
(\mathrm{m} / \mathrm{s})\end{array}$ & $\begin{array}{c}\text { Re } \\
(\mathrm{MPa})\end{array}$ \\
\hline Gypse V1 & 15,5 & 4010 & 13,7 \\
Gypse V2A & 3,5 & 4540 & 16,9 \\
Gypse V2B & 4,6 & 4520 & 23,5 \\
\hline
\end{tabular}

Ces gypses ont été choisis pour leur homogénéité et leur faible état de fissuration que l'on peut apprécier notamment par leur indice de continuité (I) en fonction de la porosité mesurée (Fig. 1): la densité de fissuration d'une roche, exprimée en $\%$, est définie par la différence entre l'indice de continuité mesuré I et l'indice de continuité $I_{c p}$ calculé si la roche ne présente pas de porosité de fissures. Les roches dites essentiellement poreuses suivent une loi empirique $I_{\mathrm{cp}}=100-1,4 \mathrm{n}$ avec $\mathrm{n}$ la porosité de pores (CFMR, 2000). La graduation de l'espace situé sous la droite $I=100-1,4 \mathrm{n}$ permet d'apprécier qualitativement l'état de fissuration des roches étudiées (Fig. 1).

\section{3}

\section{Dispositif expérimental}

Il est constitué d'une balance d'étalonnage à fléaux dont le rapport multiplicateur des forces est de 100. L'éprouvette placée dans une enceinte remplie d'eau est comprimée verticalement entre deux plateaux (Fig. 2). Le plateau supérieur mobile, muni d'une rotule, applique l'effort. I est équipé d'un capteur de déplacement permettant la mesure de la déformation globale de l'éprouvette selon son axe (déformation axiale $\varepsilon$ ).

Pour éviter les dissolutions chiniques au cours de l'essai, l'éprouvette est placée dans une eau sursaturée en sulfates de calcium.

L'appareillage est isolé dans une pièce maintenue à la température de $13^{\circ} \mathrm{C}$ (température moyenne des carrières souterraines), pour, d'une part, empêcher les fluctuations du coefficient de dilatation de la balance et, d'autre part, pour s'affranchir du paramètre température qui intervient sur le comportement du matériau.

Le gypse V1 a servi pour du fluage longue durée (plus de 400 jours pour certaines éprouvettes) avec chargement unique; tandis que le gypse V2 a été utilisé en fluage par paliers et les essais d'une durée totale comprise entre 80 à 300 jours, ont été arrêtés avant la rupture afin d'observer des sections d'éprouvettes au microscope électronique à balayage (MEB), outil bien approprié pour apprécier l'état de fissuration d'un 


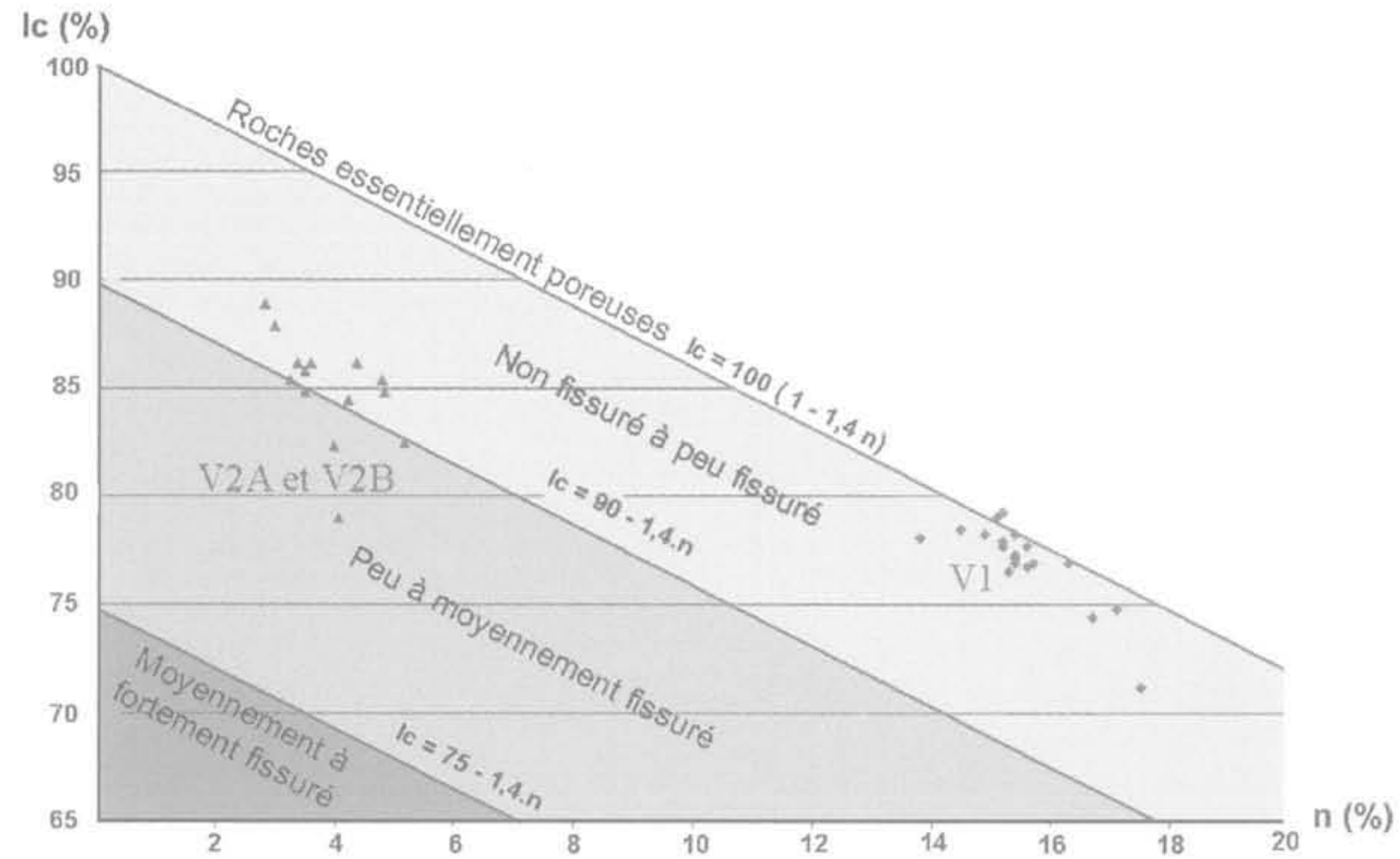

FIG.1 Diagramme Indice de continuité Ic (\%) en fonction de la porosité n (\%). Diagram Index of continuity Ic $(\%)$ versus porosity $n(\%)$.

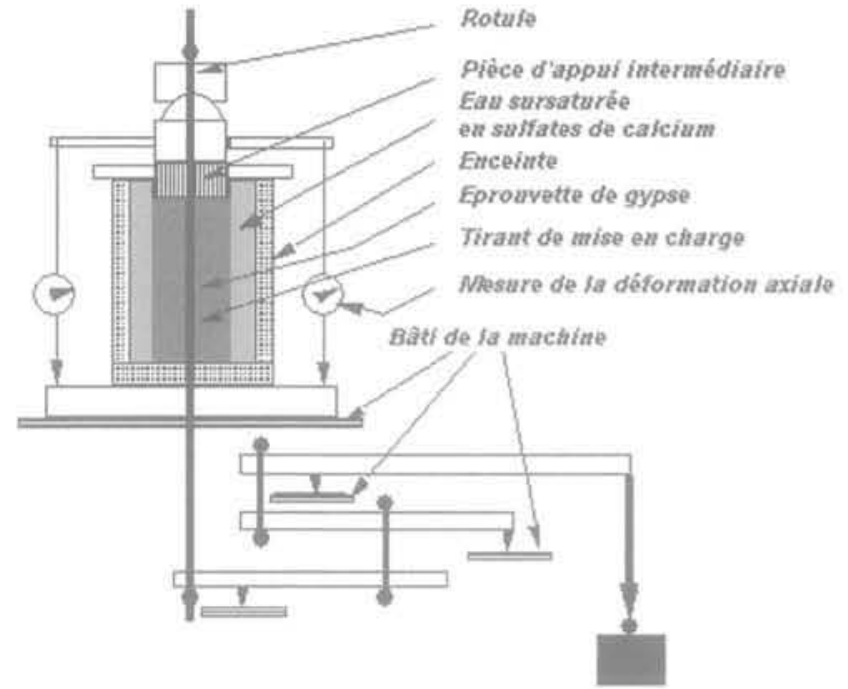

FIG.2 Schéma du dispositif expérimental. Diagram of the experimental device. matériau. Les valeurs des chargements appliqués sont données dans le tableau II.

\section{Résultats expérimentaux}

\section{1}

\section{Comportement du gypse lors du fluage}

Pour une contrainte appliquée $\sigma$, on observe (Figs. 3 et 4):

- Lorsque $0<\sigma \leq 33 \%$ de $\mathrm{R}_{c \mathrm{w}}$ (gypse V1) ou $0<0$ $\leq 55 \%$ de $\mathrm{R}_{\mathrm{cov}}$ (gypse V2), une phase de fluage transitoire très courte d'une durée de l'ordre de 20 heures: suivie par du fluage lent, asymptotique, caractérisé par une relation pseudo linéaire entre la déformation et le temps (éprouvettes V1-9, Fig. 3; et éprouvettes V2A-11, Fig. 4).

Le seuil expérimental de $33 \%$ de $\mathrm{R}_{\mathrm{cw}}$ (gypse V1) ou

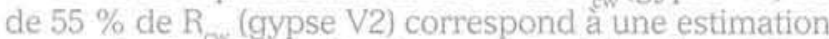
par excès de la limite élastique $\sigma$ selon Tincelin et Tijani (1982): tant que la contrainte est inférieure ou égale à la limite élastique, le fluage se stabilise avec le temps (Mor-

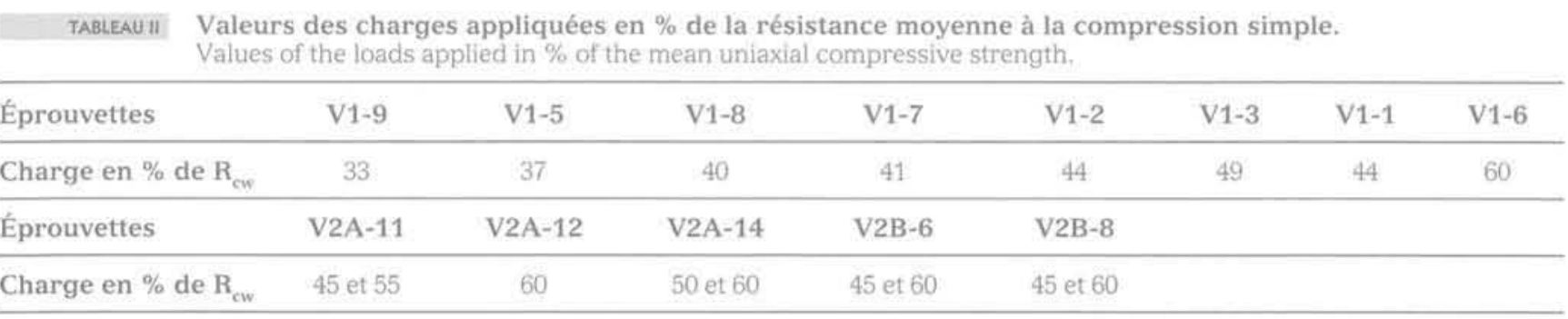


lier, 1966). La valeur de $33 \%$ de $R_{\text {w }}$ pour le gypse V1 est cohérente avec la gamme de valeurs de seuil de foisonnement de 20 à $40 \%$ de $R_{\text {cú }}$ obtenues sur des gypses cristallins de la butte de l'Hautil (même origine que le gypse V1) testés en compression simple et en condition saturée (Massieu et Martinet, 1996). En effet on détermine généralement la limite élastique lors d'un essai en compression simple, la valeur de cette limite étant donnée par le seuil de foisonnement (CFMR, 2000) qui est la limite entre les domaines de propagation de la fissuration stable et instable (instable au sens où si l'on arrête de monter en charge la fissuration continue de progresser). Mais ce seuil de foisonnement est également une valeur par excès de la limite élastique, la valeur obtenue lors d'un essai de fluage étant plus proche de la valeur vraie de part la lenteur de l'essai (Tincelin et Tijani, 1982). Seulement, une estimation précise de la limite élastique demanderait des essais trop longs pour être entreprise.

- Lorsque $\sigma>33 \%$ de $\mathbf{R}_{c w}$ (gypse V1) ou $\sigma>55 \%$ de $R_{c w}$ (gypse V2), et après la phase rapide du fluage transitoire, le fluage est caractérisé par une relation pseudo-linéaire entre la déformation et le temps. Plus la contrainte appliquée est élevée, plus la pente $(\Delta \varepsilon / \Delta t)$ est forte (exemple du gypse V1, Fig. 3).

A partir d'une valeur $\varepsilon_{0}$ de la déformation, le fluage s'accélère: la pente de la courbe déformation en fonction du temps $(\Delta \varepsilon / \Delta t)$ s'accroît rapidement. L'éprouvette prend alors la forme classique du tonneau du fait du frettage aux contacts plateau/éprouvette. Cela a été bien observé pour le gypse V1 (Fig. 3) soumis à charge constante, $\varepsilon_{0} \approx 5.10^{-3}$ pour l'éprouvette V1-5, et $\varepsilon_{0} \approx 3$, $7.10^{-3}$ pour les éprouvettes V1-7 et V1-8; et moins précisément pour le gypse V2 (Fig. 4) soumis à un charge- ment par paliers, $\varepsilon_{0} \approx 8,5.10^{-3}$ (éprouvettes V2B6, V2B8).

Au voisinage de la valeur $\varepsilon_{0 \gamma}$ apparaissent au centre de l'éprouvette des lignes de cisaillement conjuguées pour le gypse V1 (analogues aux lignes de Lüders en métallurgie, information orale de V. Maury), beaucoup moins marquées pour le gypse V2 plus résistant que pour le gypse V1. Ces lignes évoluent en fissures dont l'une généralement progresse en empruntant un des plans tangents aux deux cônes de frettage. On passe ainsi au voisinage de $\varepsilon_{0}$ d'un mode de déformation continu (sans fissuration visible à l'ceil nu) à un mode discontinu, ce qui est analogue au comportement en fluage du sel gemme dont le mode de cristallisation par accrétion est similaire au gypse (Chen et Wang, 1993; Chen et al., 1997; Yahya et al., 2000).

- Lorsque $\sigma \geq 60 \%$ de $\mathrm{R}_{c w}$ (gypse V1), il n'y a pas de stade de fluage transitoire ou de stade de fluage caractérisé par une relation pseudo linéaire entre la déformation et le temps. La déformation est rapide et l'éprouvette va rapidement à la rupture (éprouvette V1-6, Fig. 4);

On observe un ou deux stades de fluage suivant la valeur de la contrainte appliquée o par rapport à la valeur de la limite élastique $\sigma_{1}$ :

- si $\sigma \leq \sigma_{\gamma}$ le fluage, caractérisé par une relation pseudolinéaire entre la déformation et le temps (stade de fluage stationnaire) est asymptotique: la déformation tend vers une constante,

- si $\sigma>\sigma_{y}$ le fluage est caractérisé pour $\varepsilon<\varepsilon_{0}$ par une relation pseudo-linéaire entre la déformation et le temps (stade de fluage stationnaire); et pour $\varepsilon \geq \varepsilon_{0}$, par une accélération de la déformation jusqu'à la rupture brutale et rapide de l'éprouvette (stade de fluage accéléré).

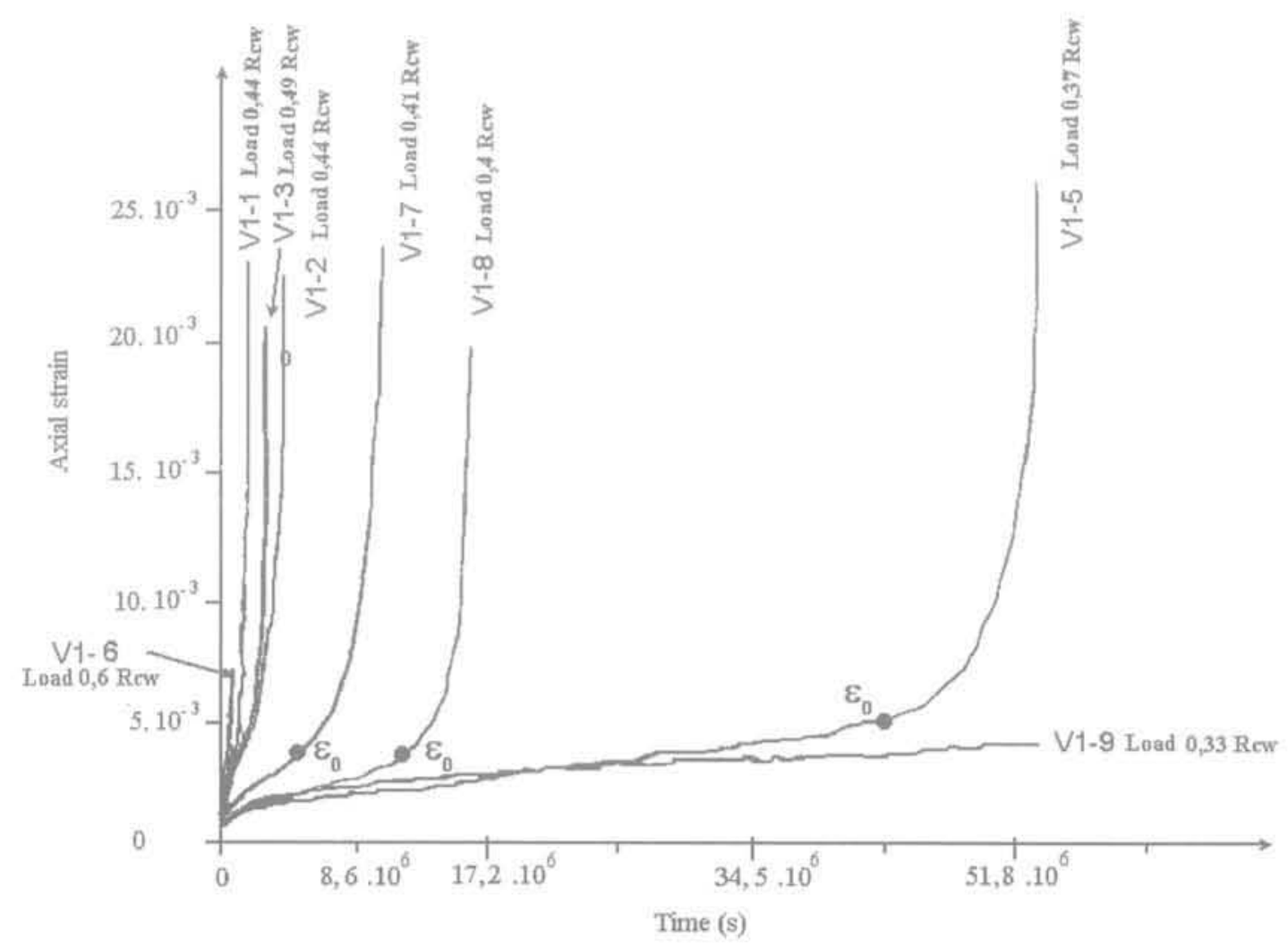

FG. 3 Courbes de la déformation axiale en fonction du temps pour le gypse V1. Axial strain versus time curves for V1 gypsum. 

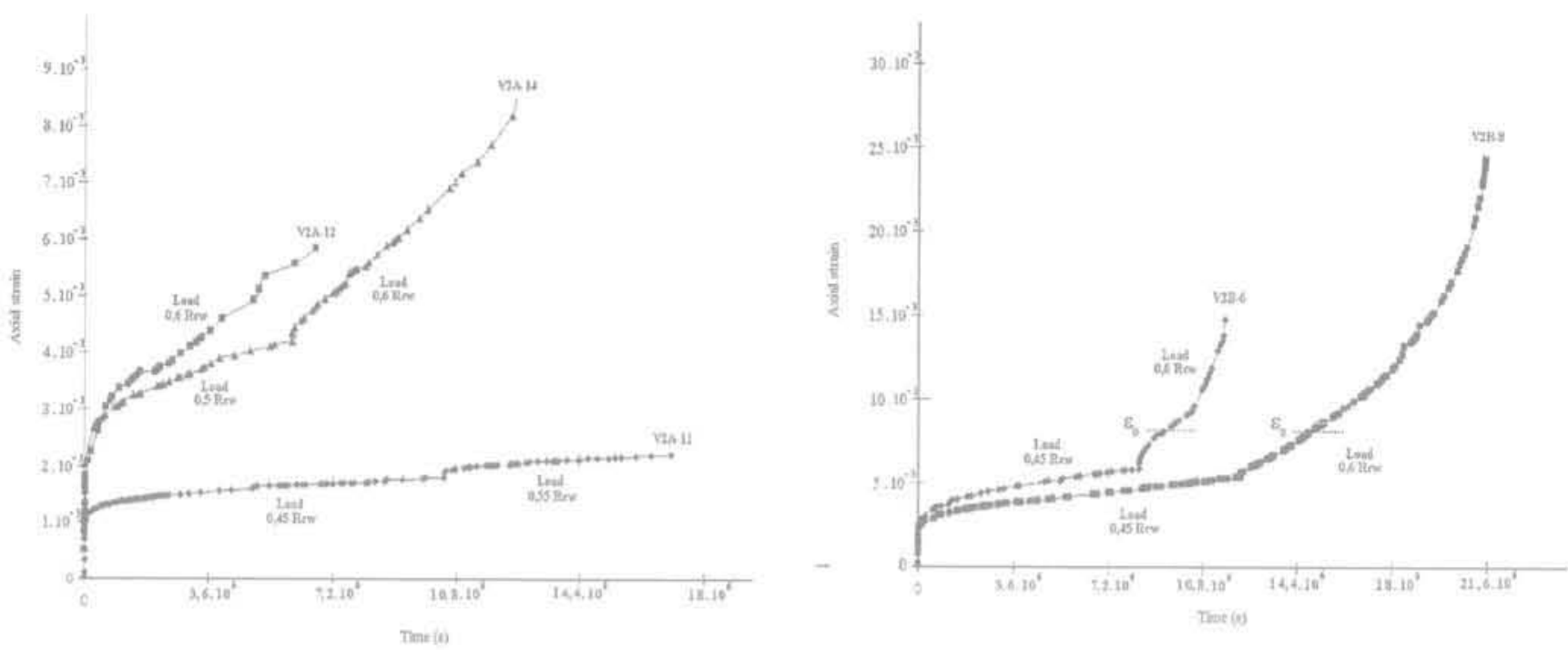

F1G. 4. Courbes de la déformation axiale en fonction du temps pour le gypse V2A et V2B. Axial strain versus time curves for the V2A and V2B gypsum.

\section{Relation entre la vitesse de déformation et le temps de rupture $t_{r}$ de l'éprouvette (gypse V1)}

Lors du fluage dit stationnaire et non asymptotique $\left(\sigma>\sigma_{1}\right)$, les courbes vitesses de déformation $(\Delta \varepsilon / \Delta t)$ en fonction du temps sur une échelle logarithmique, montrent que, pour chaque éprouvette la vitesse de déformation n'est pas constante mais décroit faiblement et régulièrement jusqu'à atteindre une valeur seuil minimale $V_{0}$ aut-delà de laquelle démarre le fluage accéléré (Fig. 5), Cependant, pour l'éprouvette V1-5 (essai le plus long), la vitesse de déformation, après avoir baissé régulièrement, s'est stabilisée au voisinage de $4.10^{-11} \mathrm{~s}^{-1}$ sur plus de 300 jours avant que ne s'amorce le fluage accéléré.

Pour les éprouvettes de gypse V1 sur lesquelles la déformation $\varepsilon_{0}$ a pu être mesurée (éprouvettes V1-5, V1-7 et V1-8), le produit de la vitesse minimale $V_{0}$ par le temps de rupture t, mesuré depuis le début du chargement, avoisine la moitié de la déformation $\varepsilon_{0}$ (Tableau III):

$$
\mathrm{V}_{0} \cdot \mathrm{tr} \approx \varepsilon_{0} / 2
$$

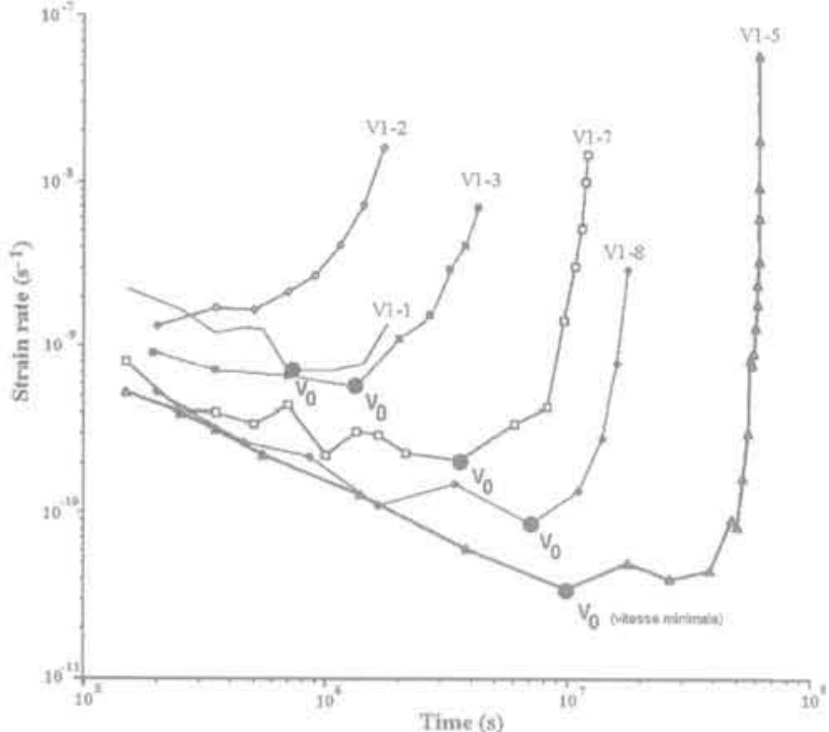

Fig.5 Courbe de la vitesse de déformation en fonction du temps pour le gypse V1. Strain rate variation versus the time for the V1 gypsum.

TABLEAUIII Comparaison des valeurs de $\left(\mathrm{V}_{0}, \mathrm{t}_{\mathrm{r}}\right)$ et de $\left(\varepsilon_{0} / 2\right)$ pour les éprouvettes V1-7, V1-8 et V1-5, avec $\mathrm{V}_{0}$ la vitesse minimale mesurée lors du fluage, $t$ le temps de rupture mesuré depuis le début du chargement et $\varepsilon_{0}$ la déformation axiale au-delà de laquelle le fluage accéléré démarre.

Comparison between the values of $\left(\mathrm{V}_{0}, \mathrm{t}\right)$ and $\left(\varepsilon_{0} / 2\right)$ for $\mathrm{V} 1-7, \mathrm{~V} 1-8$ and $\mathrm{V}_{1}-5$; being $\mathrm{V}_{0}$ the minimal strain rate measured during creep, tr the time of rupture measured since the beginning of the loading and $\varepsilon_{\mathrm{g}}$ the axial deformation beyond which accelerated creep starts.

\begin{tabular}{|c|c|c|c|c|c|c|}
\hline & $\begin{array}{l}\text { Charge } \\
\left(\% R_{c w}\right)\end{array}$ & $\begin{array}{c}V_{0} \\
\left(s^{-1}\right)\end{array}$ & $\begin{array}{l}t_{r} \\
(s)\end{array}$ & $\varepsilon_{0}$ & $V_{o} \cdot t_{r}$ & $\varepsilon_{0} / 2$ \\
\hline V1-7 & 41 & $2,2.10^{-30}$ & $9,2 \cdot 10^{6}$ & $3,75.10^{-2}$ & $2,02 \cdot 10^{-3}$ & $1,87 \cdot 10^{-3}$ \\
\hline V1-8 & 40 & $1.10^{-10}$ & $1,6.10^{7}$ & $3,75.10^{-3}$ & $1,66 \cdot 10^{-9}$ & $1,87.10^{-3}$ \\
\hline V1-5 & 37 & $5.10^{-11}$ & $5,2.10^{7}$ & $5.10^{-3}$ & $2,6.10^{-3}$ & $2,5.10^{-3}$ \\
\hline
\end{tabular}




\section{Aspect microscopique du mécanisme de rupture (gypse V2)}

Les essais sur les éprouvettes du faciès V2 ont été arrêtés à différents états de fluage (Fig. 3): fluage stationnaire asymptotique (V2A-11), fluage stationnaire non asymptotique (V2A-12) et fluage accéléré (V2A-14). Les caractéristiques expérimentales aux arrêts d'essais sont indiquées sur le tableau IV.

TABLEAU IV Valeurs de la déformation axiale $\varepsilon$ et de la vitesse de déformation axiale $(\Delta \varepsilon / \Delta t)$ à l'arrêt de l'essai pour les éprouvettes V2A-11, V2A-12, V2A-14.

Values of the applied load, axial strain $\varepsilon$ and strain rate $(\Delta E / \Delta t)$ at the end of the test for the gypsum V2A-11, V2A-12, V2A-14.

\begin{tabular}{|c|c|c|c|c|}
\hline & $\begin{array}{c}\text { Durée de l'essai } \\
\text { (s) }\end{array}$ & $\begin{array}{l}\text { Charge } \\
\left(\% \text { de } \mathrm{R}_{(w)}\right)\end{array}$ & $\varepsilon$ & $\begin{array}{c}\Delta \varepsilon / \Delta t \\
\left(\mathrm{~S}^{-1}\right)\end{array}$ \\
\hline V $2 A-11$ & $1,7.10^{?}$ & 55 & $2,2.10^{-3}$ & $3.10^{-11}$ \\
\hline $\mathrm{V} 2 \mathrm{~A}-12$ & $6,6.10^{6}$ & 60 & $5,8.10^{-3}$ & $4,5.10^{-16}$ \\
\hline $\mathrm{V} 2 \mathrm{~A}-14$ & $1,2.10^{7}$ & 60 & $8.1 .10^{-3}$ & $5,6.10^{-10}$ \\
\hline
\end{tabular}

Ces trois éprouvettes ont été ensuite enrobées dans une résine époxy afin de maintenir au mieux l'état des structures lors du découpage de sections. Douze coupes à la scie diamantée très fine ont été réalisées, parallèlement (1 coupe par éprouvette) et perpendiculairement ( 3 coupes par éprouvette) à la direction de la contrainte appliquée $\sigma$. Ces coupes, longitudinales ou transversales, passent au voisinage du centre des éprouvettes là où l'influence du frettage est moindre. Ces douze sections ont été ensuite métallisées puis observées au MEB (9 images par section d'éprouvettes, dont 3 images par échelle d'observation 700, 500 ou $200 \mu \mathrm{m})$.

Dans ces conditions expérimentales réduites:

- le stade de fluage stationnaire asymptotique, comparé à des échantillons témoins de gypse V2A (Potherat et al., 1999), se traduit essentiellement par une décohésion légère entre les grains (V2A-11, Fig. 6);

- puis, lors du stade de fluage stationnaire non asymptotique (V2A-12, Fig. 6) cette décohésion intergranulaire s'accroît et s'accompagne d'une microfissuration se développant le long de plusieurs grains, et prépondérante dans les plans perpendiculaires à la direction de la contrainte

- enfin, lors du stade de fluage accéléré (V2A-14, Fig. 6), cette décohésion et cette microfissuration se densifient et s'amplifient (longueurs des fissures et ouvertures entre les grains plus importantes qu'au stade précédent). Certains grains sont fragmentés et leur taille moyenne (non mesurée) s'est réduite.

Les observations de déstructuration progressive lors du fluage stationnaire à accéléré vont dans le sens de l'augmentation volumique observée macroscopiquement (forme en tonneau de l'éprouvette frettée), mais non mesurée (pas de capteurs de déplacements mesurant la déformation transversale lors des essais)

Par ailleurs, pour une échelle donnée $(700,500$ ou $200 \mu \mathrm{m})$, le seuillage d'une image MEB en noir et blanc puis le traitement de cette image (comptabilité des pixels noirs (vide) et blancs (zone granulaire)) permet de calculer une porosité relative moyenne pour chaque image, somme de la porosité de pores et de fissures.

A l'échelle de $500 \mu \mathrm{m}$, trois images MEB par section d'éprouvettes, chacune perpendiculaire à la direction de la contrainte et passant par le centre des éprouvettes, ont été traitées. Les résultats, qui demandent à être confirmés par un plus grand nombre d'images traitées ainsi que par des essais complémentaires, montrent qu'il existe un saut de porosité moyenne entre le fluage stationnaire et le fluage accéléré : en effet, pour le faciès V2A, la porosité moyenne est de $3,5 \%$ avant essai. Elle avoisine $5 \%$ pour les éprouvettes V2A-11 et V2A-12 lors du fluage stationnaire et passe à $12 \%$ au début du fluage accéléré pour l'éprouvette V2A-14.

Ainsi à la transition entre fluage stationnaire et fluage accéléré, il existerait un saut de porosité (correspondant à un seuil de fissuration) au-delà duquel l'éprouvette va inévitablement à la rupture (stade court du fluage accéléré). Ce résultat est similaire au comportement instantané d'une éprouvette soumis à un essai en compression simple où au-delà du seuil de foisonnement, la propagation de la fissuration est instable et conduit à la ruine rapide de l'éprouvette (Panet, 1976).

\section{5}

\section{Conclusion}

La texture simple du gypse saccharoïde, constitué par accolement de grains, permet de mieux appréhender les mécanismes mis en cuvre lors du fluage de ce matériau en condition saturée et sans pression de confinement.

Après un stade de fluage transitoire, il existe un ou deux stades de fluage en fonction de la valeur de la contrainte appliquée o par rapport à la valeur de la limite élastique $\sigma$ :

- si $\sigma \leq \sigma_{\gamma}$ le fluage, caractérisé par une relation pseudo-lineaire entre la déformation et le temps (stade de fluage stationnaire), est asymptotique :

- si $\sigma>\sigma_{y}$ le fluage est caractérisé :

- pour $\varepsilon<\varepsilon_{0}$, par une relation pseudo-linéaire entre la déformation et le temps (stade de fluage stationnaire). Lors de ce stade, la vitesse de déformation axiale $(\Delta \mathrm{e} / \Delta \mathrm{t})$ tend vers une valeur minimale $V_{0}$ et le produit de cette vitesse minimale $V_{0}$ par le temps de rupture $t_{\text {, }}$ mesuré depuis le début du chargement, avoisine $\varepsilon_{d} / 2$ (relation déjà mentionnée par Morlier, 1966);

- pour $\varepsilon \geq \varepsilon_{0}$, par une accélération de la déformation jusqu'à la rupture brutale et rapide de l'éprouvette (stade de fluage accéléré).

Les observations au MEB, qui sont à confirmer par un plus grand nombre d'images et d'essais complémentaires, tendent à montrer que le mécanisme de rupture, lors du fluage, se caractérise par une décohésion des grains puis par microfissuration croissante et une fragmentation des grains. Cette déstructuration du matériau se traduirait par une augmentation de la porosité relative, progressive lors du fluage stationnaire, puis brutale au début du fluage accéléré qui conduit à la ruine rapide et brutale de l'éprouvette. Ce saut brusque de porosité au début du fluage accélêré, soit 

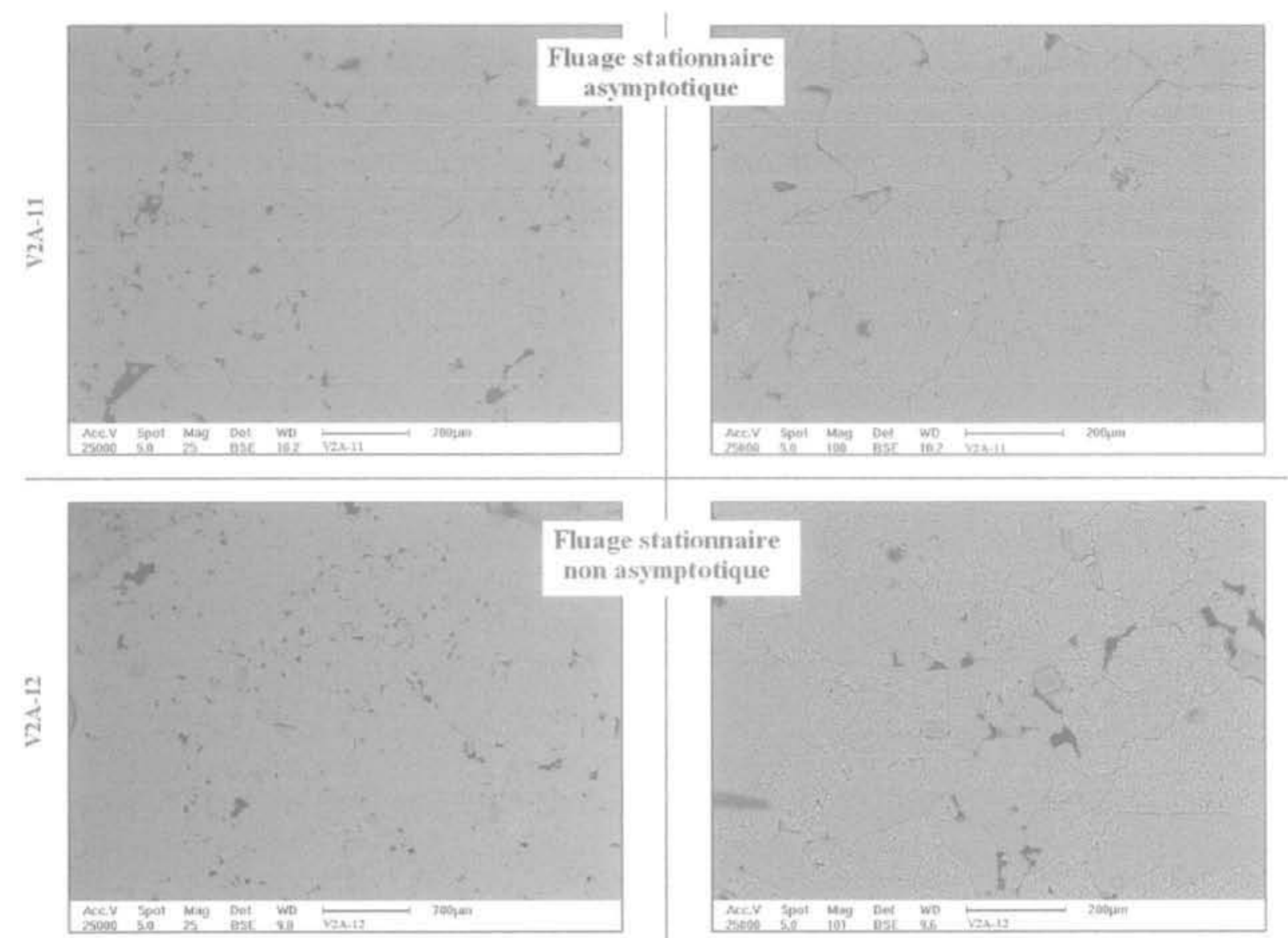

Fluage stationnaire non asymptotique

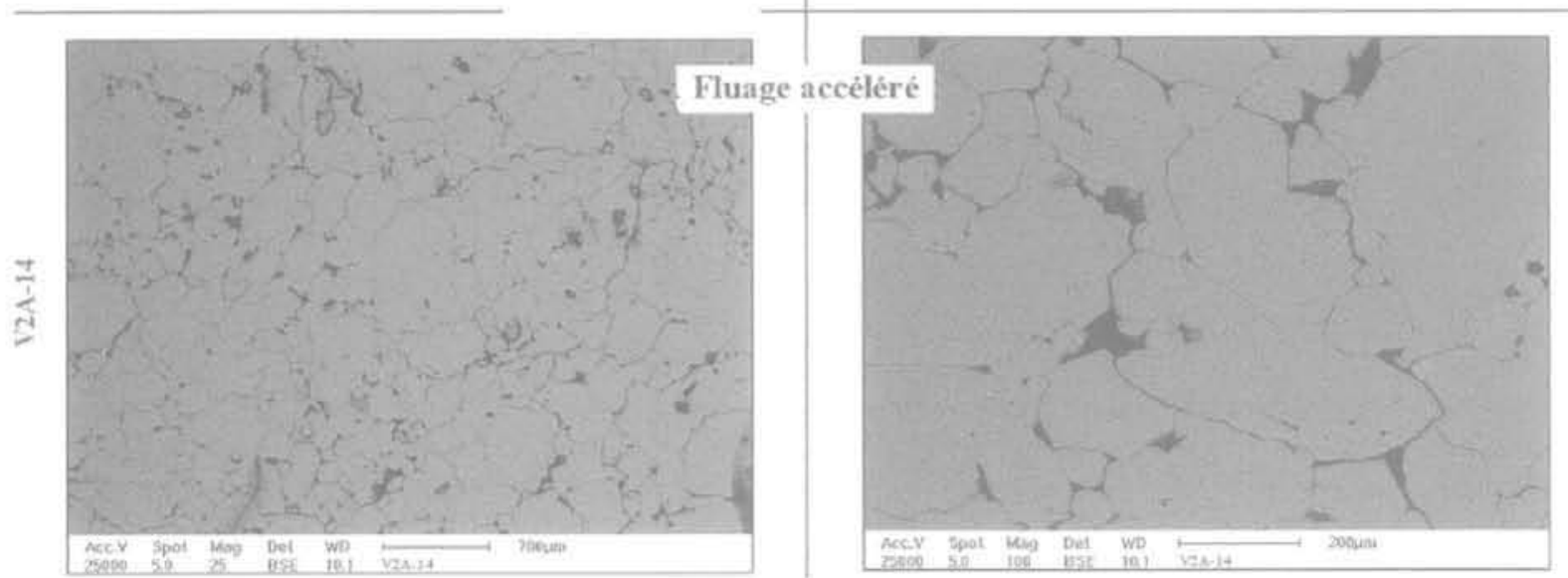

FG.6 Aspect microscopique du fluage à deux échelles (images MEB $700 \mu \mathrm{m}$ et $200 \mu \mathrm{m}$ ). Sections au centre des éprouvettes et perpendiculaíres à la dírection de la contrainte appliquée.

Microscopic aspect of creep on two scales (images MEB $700 \mu \mathrm{m}$ and $200 \mu \mathrm{m}$ ). Sections in the center and perpendicular to the direction of the applied stress.

au voisinage de la déformation $\varepsilon_{\rho}$ est en concordance avec les observations macroscopiques du passage d'un mode de déformation continu (sans fissuration visible à l'œil nu) à un mode discontinu.

Ces différents résultats ne sont malheureusement pas directement transposables aux piliers des carrières souterraines soumis à charge constante, du fait:

- de l'effet d'échelle et de l'effet de forme (pilier trapézoïdal ou rectangulaire):

- des différents types de gypse saccharoïde constituant un pilier (une taille moyenne de grain par banc de gypse et une épaisseur pour chaque banc)
- des variations hygrométriques qui influent nettement sur les vitesses de fluage (Auvray et al., 2004);

- et de P'anisotropie du gypse (toutes les éprouvettes ont été taillées perpendiculairement au litage).

Néanmoins, ces résultats peuvent être exploités, en termes de calage de modèles rhéologiques dont l'introduction dans des codes numériques adéquats permettrait une meilleure analyse de la stabilité à long terme des carrières souterraines, Enfin, les observations sur les mécanismes de ruptures montrent que dans un pilier, des bancs de gypse apparemment sains (sans fissuration macroscopique visible) peuvent être en fait relativement dégradés, proches du stade de fluage accéléré et donc de la ruine. 
Durville J.- L Massieu E., Martineau F, Étude physico-mécanique du gypse d'une carrière souterraine en région parisienne. Geotechnical Engineering of Hard Soils-Softs Rocks, Symposium AlGI, Athens, Greece, 20-23 sept. 1993.

Auvray C., Homand F., Hoxha D., Didier C. - Influence du temps et de l'hygrométrie sur le comportement du gypse. Revue française de géotechnique 106107, 2004, p. 41-51

Hoxha D., Giraud A., Homand F. Modelling long-term behaviour of a natural gypsum rock. Mechanics of Materials, vol. 37-12, 2005, p. 1223 1241.

Deicha G. - Fluctuations cristallogênètiques et séquences évolutives dans le gypse parisien. Bull. inf: géo, bassin parisien 41, 2004, p. 15-17.

Potherat P., Durville J.-L., Guédon S., Massteu E. et Martinet L.. Etude de la stabi. lité d'une carrière de gypse de la butte de
Montmorency, aspects géologiques et géomécaniques. Rapport interne LCPC Activité n $327206,1999,44$ p.

Fourmaintraux D. - Caractérisation des roches: essais de laboratoires. La mécanique des roches appliquée aux ouvrages de génie civil, Association Amicale des Ingénieurs Anciens Élèves, ENPC, 1976, p. 13-37.

Tincelin E., Tijani S.M. - La stabilité dé la surface à l'aplomb des exploitations souterraines en mines métalliques ou assimilées. Rev. ind, min. Les Techniques 1982, p. 2-82.

Morlier P. - Le fluage des roches. Ann. ind, tech batt, trav pub. 217, 1966, p. 90 112.

Massieu E., Martinet L. - Essais triaxiaux sur du gypse provenant d'une carrière souterraine de la région parisienne. Sujet 224246 , commission technique 24, rapport interne LCPC. déc. 1996 . $15 \mathrm{p}$.
CFMR - Manuel de mécanique des roches, Presses de l'École des mines de Paris, tome 1, Fondements, 2000, $280 \mathrm{p}$.

Chen Z. Wang M.L. - A micro and macromechanical modeling of creep mecha nisms for the WIPP rock sait. Proc 3 rd Annu. WERC Tech. Devel. Conf, New Mexico Waste-Mamt. Educ, and Res. Consortium (WERC), Las Cruces, N. M. 1993, p. 23-33

Chen Z,, Wang M.L., Lu T. - Study of tertiary creep of rock salt. J. Eng. Mech., ASCE, 123 (1), 1997, p. 233-242.

Yahya O.M.L., Aubertin M. Julien M.R. A unified representation of the plasticity, creep and relaxation behaviour of rock salt, Int, J. Rock Mech. Min. Sct. And Geomech. 37, 2000, p. 787-800.

Panet M. - Les propriétés mécaniques des roches. La mécanique des roches appliquée aux ouvrages de génie civil. Association Amicale des Ingénieurs Anciens Eléves, ENPC. 1976, p. 13-37. 